\title{
gp130 in late osteoblasts and osteocytes is required for PTH- induced osteoblast differentiation
}

\author{
Therese Standal, ${ }^{1,3}$, Rachelle W Johnson', Narelle E McGregor', Ingrid J Poulton', \\ Patricia W M Ho', T John Martin ${ }^{1,2}$ and Natalie A Sims ${ }^{1,2}$ \\ ${ }^{1}$ St.Vincent's Institute of Medical Research, 9 Princes St, Fitzroy, Victoria 3065, Australia \\ ${ }^{2}$ Department of Medicine at St. Vincent's Hospital Melbourne, The University of Melbourne, \\ Fitzroy, Victoria, Australia \\ ${ }^{3}$ Department of Cancer Research and Molecular Medicine, The KG Jebsen Center for Myeloma Research and Centre \\ of Molecular Inflammation Research, Norwegian University of Science and Technology, Trondheim, Norway
}

Correspondence should be addressed to N A Sims

Email

nsims@svi.edu.au

\begin{abstract}
Parathyroid hormone (PTH) treatment stimulates osteoblast differentiation and bone formation, and is the only currently approved anabolic therapy for osteoporosis. In cells of the osteoblast lineage, PTH also stimulates the expression of members of the interleukin 6 (IL-6) cytokine superfamily. Although the similarity of gene targets regulated by these cytokines and PTH suggest cooperative action, the dependence of PTH anabolic action on IL-6 cytokine signaling is unknown. To determine whether cytokine signaling in the osteocyte through glycoprotein 130 (gp130), the common IL-6 superfamily receptor subunit, is required for PTH anabolic action, male mice with conditional gp130 deletion in osteocytes (Dmp1Cre.gp130 f/f) and littermate controls (Dmp1Cre.gp130w/m) were treated with $\mathrm{hPTH}(1-34)(30 \mu \mathrm{g} / \mathrm{kg} 5 \times$ per week for 5 weeks). PTH dramatically increased bone formation in Dmp1Cre.gp130 w/w mice, as indicated by elevated osteoblast number, osteoid surface, mineralizing surface, and increased serum N-terminal propeptide of type 1 collagen (P1NP). However, in mice with Dmp1Cre-directed deletion of gp130, PTH treatment changed none of these parameters. Impaired PTH anabolic action was associated with a $50 \%$ reduction in Pth1r mRNA levels in Dmp1Cre.gp130 f/f femora compared with Dmp1Cre.gp130w/w. Furthermore, lentiviral-Cre infection of gp130 f/f primary osteoblasts also lowered Pth1r mRNA levels to $16 \%$ of that observed in infected C57/BL6 cells. In conclusion, osteocytic gp130 is required to maintain PTH1R expression in the osteoblast lineage, and for the stimulation of osteoblast differentiation that occurs in response to PTH.
\end{abstract}

$\begin{aligned} & \text { Key Words } \\ & \text { - glycoprotein-130 (gp130) } \\ & \text { - osteoblast } \\ & \text { - osteocyte } \\ & \text { - osteoclast } \\ & \text { - PTH } \\ & \text { - PTH1R } \\ & \text { - trabecular } \\ & \text { - cortical } \\ & \text { - bone formation }\end{aligned}$

\section{Introduction}

Intermittent administration of parathyroid hormone (PTH) to animal models and humans (teriparatide (Forteo)) increases bone mass (Reeve et al. 1980, Neer et al. 2001, Lindsay et al. 2007), and is the only approved treatment for osteoporosis capable of inducing bone formation (reviewed in Hodsman et al. (2005) and Khosla et al. (2008)). However, the mechanisms by which intermittent PTH increases bone mass remain unclear, and identifying downstream targets of this pathway may aid in the design of improved anabolic therapies.

Published by Bioscientifica Ltd. 
The effects of PTH on bone mass are likely to be mediated by cells of the osteoblast lineage. This lineage includes committed pre-osteoblasts, matrix-producing osteoblasts, bone lining cells, and matrix-embedded osteocytes. PTH acts directly at each stage of differentiation, as follows. PTH promotes pre-osteoblast differentiation (Dobnig \& Turner 1995), inhibits osteoblast apoptosis (Jilka et al. 1999), and reactivates quiescent lining cells to become active osteoblasts (Kim et al. 2012). PTH also acts directly on osteocytes to reduce their expression of the WNT antagonist sclerostin, an inhibitor of bone formation (Bellido et al. 2005, Keller \& Kneissel 2005).

PTH also stimulates the expression of receptor activator of NF-kappa-B ligand (RANKL) by early osteoblast lineage cells, thereby promoting osteoclast differentiation (Udagawa et al. 1999). However, the stages of osteoblast differentiation most important for the actions of PTH remain controversial, because the expression of RANKL by matrix-embedded osteocytes is also stimulated by PTH (Xiong et al. 2011).

PTH also acts on the osteoblast lineage to rapidly promote the transcription of interleukin 6 (IL-6) family cytokines and receptors. These include Il6 (Greenfield et al. 1996), Il11, oncostatin M receptor (Osmr), leukemia inhibitory factor ( $L i f)$, and cytokine receptor-like factor 1 (Crlf1) (Walker et al. 2012). These cytokines all depend on the promiscuous co-receptor glycoprotein 130 (gp130) for signaling (reviewed in Sims \& Walsh (2010)), and gp130 expression by the osteoblast lineage is also stimulated by PTH (Romas et al. 1996).

Many of the actions and gene targets of IL- 6 family cytokines are common to those of PTH. As is the case with PTH, the cytokines IL-6, IL-11, OSM, LIF, and cardiotrophin (CT-1) promote osteoblast differentiation in vitro (Walker et al. 2008, 2010) and OSM, LIF, and CT-1 stimulate bone formation in vivo (Cornish et al. 1993, Walker et al. 2008, 2010). The family members IL-11, LIF, OSM, CT-1, and CNTF also inhibit osteocytic sclerostin expression (Walker et al. 2010, Johnson et al. 2014a). In addition, IL-6, IL-11, OSM, LIF, and CT-1, stimulate osteoblast lineage expression of RANKL $\left(\mathrm{O}^{\prime}\right.$ Brien et al. 1999, Palmqvist et al. 2002, Walker et al. 2008) and promote osteoclastogenesis when precursors are co-cultured with osteoblasts in vitro (Tamura et al. 1993, Richards et al. 2000). These similar effects and the upregulation of IL-6 family cytokines in osteoblasts by PTH suggest that this cytokine family may play a role in the actions of PTH on the osteoblast lineage.

Hence, in this study we examined the requirement of gp130 signaling in osteocytes for the anabolic action of
PTH, using mice with Dmp1Cre-directed deletion of gp130 in osteocytes (Dmp1Cre.gp130 fff; Johnson et al. 2014b) and mature osteoblasts (Xiong et al. 2011, Torreggiani et al. 2013). We found that gp130 in these cells is required for PTH to increase osteoblast number and bone forming surfaces, and to maintain PTH1R expression in the osteoblast lineage.

\section{Materials and methods}

\section{Mice}

All animal procedures were conducted with the approval of the St. Vincent's Health Melbourne Animal Ethics Committee. Dmp1Cre mice were obtained from Lynda Bonewald (University of Kansas, Kansas City, MO, USA; Lu et al. 2007). Floxed gp130 mice backcrossed onto C57/BL6 were obtained from Rodger McEver (Oklahoma Medical Research Foundation, Oklahoma City, OK, USA; Betz et al. 1998). Mice hemizygous for the Cre transgene were crossed with the gp130 flox mouse in which the transmembrane domain (exon 15) was flanked by loxP sites, resulting in ablation of intracellular gp130 signaling, as previously reported (Betz et al. 1998) and confirmed at the mRNA level in bone (Johnson et al. 2014b). For all experiments, Dmp1.Cre + cousins were used as controls.

Six-week-old male Dmp1Cre.gp130 1 w/w or floxed Dmp1Cre.gp130 f/f mice were injected i.p. with $30 \mu \mathrm{g} / \mathrm{kg}$ human PTH 1-34 (hPTH 1-34) or vehicle, 5 days a week for 5 weeks ( $n=9 / 10$ per group). This dose and duration of PTH treatment were chosen because it provides a robust increase in lamellar bone formation rate and osteoblast surface in male mice without increasing osteoclastogenesis (Walker et al. 2012, Takyar et al. 2013, Tonna et al. 2014). The mice were also injected with calcein $(20 \mathrm{mg} / \mathrm{kg}) 7$ and 2 days before tissue collection. The bones were collected $1 \mathrm{~h}$ after the last PTH injection. The mice were fasted for $12 \mathrm{~h}$ before anaesthesia with ketamine/ xylazine and a final blood sample was collected by cardiac puncture. The blood samples were centrifuged for $10 \mathrm{~min}$ at $4000 \mathrm{~g}$ and the serum was collected in a fresh tube and stored at $-80^{\circ} \mathrm{C}$ until analysis for cross-linked C-terminal telopeptide of type 1 collagen (CTX1), N-terminal propeptide of type 1 collagen (P1NP) (Immunodiagnostic Systems Limited, Boldon, Tyne and Wear, UK), and PTH (Immunotopics, San Clemente, CA, USA) as per manufacturer's instructions. One femur was flushed of marrow and the bone shaft was collected for RNA analyses as described previously (Walker et al. 2012). Briefly, bones were homogenized with a LS-10-35 Polytron homogenizer in

Published by Bioscientifica Ltd. 
TRI for $4 \times 5 \mathrm{~s}$ bursts and stored at $-80^{\circ} \mathrm{C}$. RNA from each bone was purified using the RNeasy lipid tissue minikit (Qiagen), according to manufacturer's instructions.

The other femur was analyzed by micro-computed tomography as described previously (Johnson et al. 2014b) using the SkyScan 1076 System (Bruker-microCT, Kontich, Belgium). The images were acquired using the following settings: $9 \mu \mathrm{m}$ voxel resolution, $0.5 \mathrm{~mm}$ aluminium filter, $48 \mathrm{kV}$ voltage, and $100 \mu \mathrm{A}$ current, exposure time, rotation $0.5^{\circ}$, frame averaging $=1$. The images were reconstructed and analyzed using SkyScan Software programs NRecon (version 1.6.3.3), DataViewer (version 1.4.4), and CT Analyser (version 1.12.0.0). Femoral trabecular analysis region of interest (ROI) was determined by identifying the distal end of the femur and calculating $15 \%$ of the total femur length toward the femora mid-shaft, where we then analyzed an ROI of $12.6 \%$ of the total femur length. The analysis of bone structure was completed using adaptive thresholding (mean of min and max values) in CT Analyser. The thresholds for analysis were determined based on multilevel Otsu thresholding of the entire data set, and were set at 45-255 for trabecular bone. The cortical analyses were performed at 35\% above the distal end of the femur toward the femora mid-shaft, also with a $12.6 \%$ ROI with the threshold values set at 100-255.

Tibiae were collected for histomorphometric analyses as previously described (Sims et al. 2006). Briefly, trabecular histomorphometry was carried out on undecalcified sections in the secondary spongiosa of the proximal tibia, in a region $370 \mu \mathrm{m}$ below the proximal edge of the hypertrophic zone of the growth plate, extending $1.11 \mathrm{~mm}$ in the proximal direction. Periosteal histomorphometry was carried out on the antero-fibular side of the tibia, commencing $1.11 \mathrm{~mm}$ below the chondro-osseus junction of the growth plate, and extending $1.11 \mathrm{~mm}$ in the proximal direction. The nomenclature is as described previously (Parfitt et al. 1987).

\section{Lenti-Cre viral infection}

Calvarial osteoblasts were collected from C57/BL6 WT and $g p 130^{f / f}$ neonates by digesting calvaria in $1: 2$ collagenase II/dispase solution at $37^{\circ} \mathrm{C}$ on a shaker $(1 \times 5 \mathrm{~min} 4 \times$ 10 min digestions). The cells were resuspended in culture media (alpha-MEM $+10 \%$ fetal bovine serum), and allowed to adhere overnight before being frozen and stored in liquid nitrogen. When required, isolated cells were thawed and expanded in culture and infected with a GFP-tagged lenti-Cre virus synthesized as described previously (Tonna et al. 2014) for $24 \mathrm{~h}$ with polybrene in the maintenance media. Following infection, media was changed and cells were evaluated for GFP expression by microscopy; > 30-60\% transfection efficiency was observed ( $n=3$ independent experiments). The cells were expanded in culture for 2-3 weeks in alpha-MEM $+10 \%$ fetal bovine serum, and GFP positive cells (fluorescence driven by Cre transgene expression) were sorted on a FACS Aria (BD Biosciences, San Jose, CA, USA) for GFP. The GFP + cells were harvested for RNA in trizol (Life Technologies) and separated and precipitated using chloroform and isopropanol. Extracted RNA was treated with DNase using Ambion TURBO DNA-free Kit (Life Technologies) and quantified on a NanoDrop ND1000 Spectrophotemeter (Thermo Scientific, Wilmington, DE, USA).

\section{Semi-quantitative real-time PCR}

cDNA synthesis from 50 to $100 \mathrm{ng}$ DNase-treated RNA from each femur or cell culture preparation was carried out using AffinityScript (Agilent Technologies, Santa Clara, CA, USA) as per the manufacturer's instructions. The stock cDNA was diluted to a concentration of $5 \mathrm{ng} / \mu \mathrm{l}$ and semiquantitative real-time PCR (qPCR) was performed on $12.5 \mathrm{ng}$ cDNA in a reaction volume of $10 \mu \mathrm{l}$ using in-house master mix of $10 \times$ AmpliTaq Gold with SYBR Green nucleic acid gel stain (Life Technologies). Dkk1 primers were designed using NCBI Primer Blast: forward, GAGGGGAAATTGAGGAAAGC and reverse, ACGGAGCCTTCTTGTCCTTT. Other primers were as previously described for Pth1r, hypoxanthine phosphoribosyltransferase 1 (Hprt1), Sost, Tnfsf11, Il6 (Allan et al. 2008), $\beta-2$ microglobulin (B2m) (McGregor et al. 2010), and hydroxymethylbilane synthase (Hmbs) (Johnson et al. 2014b).

The samples were dispensed onto optically clear 96-well plates (Thermo Scientific) and run on a Stratagene Mx3000P (Agilent Technologies). The cycling conditions were $95{ }^{\circ} \mathrm{C}$ for $10 \mathrm{~min}\left(95^{\circ} \mathrm{C}\right.$ for $30 \mathrm{~s}, 58^{\circ} \mathrm{C}$ for $1 \mathrm{~min}$, and $72{ }^{\circ} \mathrm{C}$ for $\left.30 \mathrm{~s}\right) \times 40$ cycles, followed by a dissociation step $\left(95^{\circ} \mathrm{C}\right.$ for $1 \mathrm{~min}, 55^{\circ} \mathrm{C}$ for $30 \mathrm{~s}$, and $95^{\circ} \mathrm{C}$ for $30 \mathrm{~s}$ ). The post-run samples were analyzed using MxPro (Agilent Technologies, Santa Clara, CA, USA) and reported using linear $\triangle C T$ values normalized to the geometric mean of the two housekeeping genes (HKG) Hprt1 and Hmbs or to $B 2 m$ as indicated.

\section{Statistical analysis}

All graphs are presented as the mean/genotype+s.E.M.. $N=5-10$ animals/group as indicated in the figure legend. For in vitro experiments, data shown is the average of three

Published by Bioscientifica Ltd 
independent biological replicates. Statistical significance was considered when $P<0.05$. Differences between groups were analyzed by two-way ANOVA and post hoc Šidak multiple comparison test. Skewed variables were transformed using the natural logarithm before statistical analyses. For the lenti-viral Cre-infected primary calvarial osteoblasts, Student's $t$-test was used to assess significance. Statistical analyses were performed using GraphPad Prism version 6.0c for Mac OS X (GraphPad Software, La Jolla, CA, USA).

\section{Results}

\section{Dmp1Cre.gp130 f/f mice show no increase in the number of trabecular osteoblasts in response to PTH}

PTH treatment at $30 \mu \mathrm{g} / \mathrm{kg}$ per day significantly increased osteoblast number/bone perimeter (NOb/BPm) on trabecular bone in Dmp1Cre.gp $130^{w / w}$ mice by $76 \%$ (Fig. 1A). Osteoblast surface/bone surface (ObS/BS; Fig. 1B) and osteoid surface/bone surface (OS/BS; Fig. 1C) were also elevated by PTH treatment to similar extents. We detected no significant changes in osteoid thickness in Dmp1Cre.gp130 $0^{w / w}$ mice after PTH treatment (Fig. 1D).

In contrast to Dmp1Cre.gp $130^{w / w}$ mice, PTH treatment did not increase osteoblast or osteoid-derived parameters in age- and sex-matched Dmp1Cre.gp $130^{f / f}$ mice (Fig. 1A, B, $\mathrm{C}$ and D). Two-way ANOVA revealed that the effects of $\mathrm{PTH}$ treatment on both $\mathrm{NOb} / \mathrm{BPm}$ and $\mathrm{ObS} / \mathrm{BS}$ were significantly reduced in the Dmp1Cre.gp130f/f mice compared with Dmp1Cre.gp130 ${ }^{w / w}$ controls (interaction $P=0.039$ and $P=0.043$ respectively). This indicates that the effect of PTH on osteoblast differentiation is dependent on gp130 expression in osteocytes.

In line with the effects on osteoblast numbers, bone forming surfaces, indicated by incorporation of calcein labels, including both double-labeled surface (dLS/BS) (Fig. 1E) and single-labeled surface $(P \leq 0.05$, not shown) were significantly greater in PTH-treated Dmp1Cre.gp $130^{w / w}$ mice compared with controls. Again, this was not observed in Dmp1Cre.gp130 f// mice. Mineral apposition rate (MAR) was significantly greater in both Dmp1Cre.gp130 f/f and Dmp1Cre.gp130 $0^{w / w}$ mice treated with PTH compared with their vehicle-treated controls (Fig. 1F), indicating that an increase in mineralization rate in response to PTH is retained on those surfaces on which bone formation occurs in Dmp1Cre.gp 130 f/f mice.

PTH-treated Dmp1Cre.gp130 $0^{w / w}$ mice had significantly higher serum P1NP levels than Dmp1Cre.gp130 13 ww untreated controls. In contrast, in Dmp1Cre.gp $130^{f / f}$ mice there was no significant effect of PTH on P1NP levels
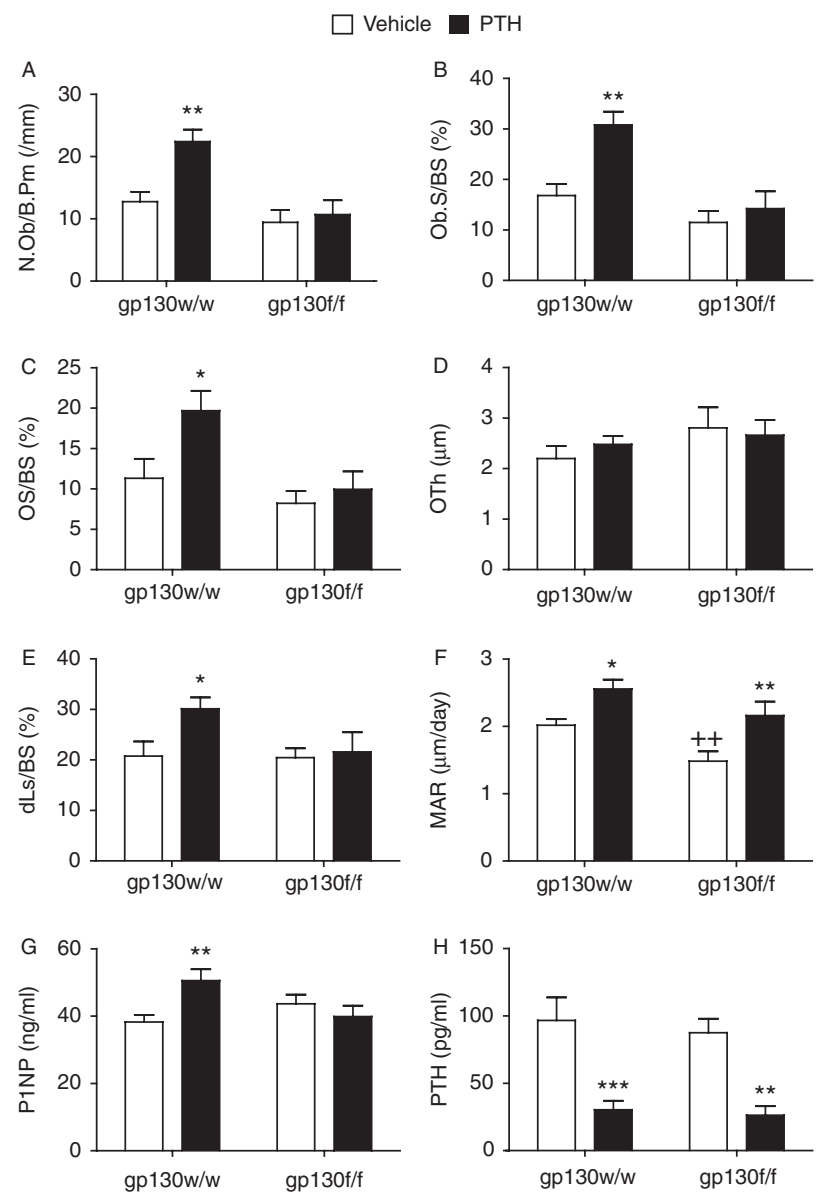

Figure 1

Osteocytic gp130 is required for PTH to increase osteoblast numbers and bone formation in trabecular bone. Male mice were treated with hPTH (1-34) at $30 \mu \mathrm{g} / \mathrm{kg}$ per day for 5 weeks. (A) Numbers of osteoblasts/bone perimeter (N.Ob/B.Pm), (B) osteoblast surface/bone surface (Ob.S/BS), (C) osteoid surface/bone surface (OS/BS), (D) osteoid thickness (OTh), (E) double calcein-labeled surface (dLS/BS), and (F) mineral apposition rate (MAR) from trabecular bone in the proximal tibial secondary spongiosa in Dmp1Cre.gp130 ${ }^{w / w}$ (gp130 w/w) and Dmp1Cre.gp130 f/f (gp130 f/f) mice. (G) The serum levels of procollagen type 1 amino-terminal propeptide (P1NP) and (H) endogenous murine PTH measured at the end of the treatment protocol are also shown. Scale bars are mean + S.E.M., $n=8-10$ per group. ${ }^{*} P \leq 0.05, * * P \leq 0.01$ and $* * * P \leq 0.001$, PTH-treated compared with genotype-matched vehicletreated mice; ${ }^{++} P<0.01$ compared to vehicle-treated gp130 w/w mice.

compared with vehicle-treated Dmp1Cre.gp130 f/f mice (Fig. 1G); interaction $P$ value $=0.009$ by two-way ANOVA. These results are consistent with the histomorphometry data and confirm that at a systemic level, the effect of PTH on bone formation is blunted in Dmp1Cre.gp $130^{f / /}$ mice.

In both Dmp1Cre.gp130 f/f and Dmp1Cre.gp130 w/w mice, intermittent human PTH treatment led to reduced production of endogenous circulating murine PTH levels (Fig. $1 \mathrm{H})$, demonstrating that negative feedback at the parathyroid gland induced by exogenous PTH administration was maintained in both groups of mice.

Published by Bioscientifica Ltd. 
Table 1 Effects of PTH on trabecular and cortical bone in femora from Dmp1Cre.gp $130^{w / w}$ and Dmp1Cre.gp $130^{w / w}$ mice.

\begin{tabular}{|c|c|c|}
\hline & \multicolumn{2}{|c|}{ Dmp1Cre.gp130w/w } \\
\hline & Vehicle $(n=9)$ & PTH $(n=10)$ \\
\hline BV/TV (\%) & $24.86 \pm 0.42$ & $21.54 \pm 1.32$ \\
\hline Tb.Th $(\mu \mathrm{m})$ & $57.61 \pm 1.80$ & $56.49 \pm 2.22$ \\
\hline Tb.N (/mm) & $4.35 \pm 0.15$ & $3.80 \pm 0.16$ \\
\hline Tb.Sp ( $\mu \mathrm{m})$ & $122.71 \pm 2.96$ & $144.39 \pm 14.04$ \\
\hline Ct.Ar $\left(\mathrm{mm}^{2}\right)$ & $0.60 \pm 0.02$ & $0.65 \pm 0.02$ \\
\hline
\end{tabular}

\begin{tabular}{|c|c|}
\hline \multicolumn{2}{|c|}{ Dmp1Cre.gp130fIf } \\
\hline Vehicle $(n=9)$ & PTH $(n=9)$ \\
\hline $17.70 \pm 1.55^{+++}$ & $20.61 \pm 0.96$ \\
\hline $57.92 \pm 3.01$ & $57.42 \pm 3.83$ \\
\hline $3.03 \pm 0.15^{+++}$ & $3.64 \pm 0.18$ \\
\hline $171.05 \pm 15.74^{+++}$ & $158.98 \pm 10.37$ \\
\hline $0.63 \pm 0.02$ & $0.67 \pm 0.03$ \\
\hline
\end{tabular}

Fixed nondemineralized femora from vehicle- or PTH-treated mice were analyzed by $\mu \mathrm{CT}$. Effect of gp $130^{\mathrm{f} / \mathrm{f}}$ transgene: ${ }^{+++} P<0.001$ vs $D$ mp $1 \mathrm{Cre.gp} 130^{w / w}$ (two-way ANOVA with Šidak multiple comparisons test). BV/TV, bone volume per total volume of the region of interest; Tb.Th, trabecular thickness; Tb.N, trabecular number; Tb.Sp, trabecular separation; Ct.Ar, cortical area.

Although this dose of PTH significantly increased all markers of bone formation in Dmp1Cre.gp130 ${ }^{w / w}$ mice, we did not detect a significant increase in trabecular bone mass by micro-computed tomography with this short-time course of low-dose treatment (Table 1). The low trabecular bone mass of these mice, previously reported (Johnson et al. 2014b), was confirmed.

\section{No effect of intermittent PTH treatment on bone resorption}

This protocol of intermittent PTH treatment did not significantly change osteoclast number/bone perimeter (NOc/BPm) (Fig. 2A), osteoclast number/osteoclast perimeter (Fig. 2B), osteoclast surface/bone surface (OcS/BS) (Fig. 2C), or serum levels of cross-linked CTX1 (Fig. 2D) in either Dmp1Cre.gp130 f/f or Dmp1Cre.gp130 $0^{w / w}$ mice. This confirms our previous observations using similar protocols over 4 weeks of treatment (Walker et al. 2012, Takyar et al. 2013, Tonna et al. 2014).

\section{Effects of PTH on cortical bone}

Periosteal MAR (Fig. 3A), periosteal mineralising surface (Fig. 3B) and periosteal perimeter (Fig. 3C) were all significantly greater in PTH-treated Dmp1Cre.gp $130^{w / w}$ mice compared with untreated mice. None of these parameters were significantly increased by PTH treatment in Dmp1Cre.gp130 f/f mice compared with genotypematched vehicle controls (Fig. 3A-C), indicating that periosteal growth in response to $\mathrm{PTH}$ may also be impaired in the absence of osteocytic gp130.

\section{Normal response of osteoclastic genes, but lack of inhibition of WNT signaling inhibitors by PTH treatment in Dmp1Cre.gp130 f/f mice}

RANKL (gene name Tnfsf11) and IL-6 (Il6) are both potent stimuli of osteoclast formation, and PTH increases their expression in cells of the osteoblast lineage (Greenfield et al. 1995, Udagawa et al. 1999). Indeed, in marrowflushed femoral samples collected $1 \mathrm{~h}$ after the last of these 5 weeks of injections, mRNA levels of Tnfsf 11 and $I l 6$ were significantly higher in both genotypes after PTH treatment (Fig. 4A and B); this increase was not significantly affected by the genotype (two-way ANOVA interaction $P$ values $=$ 0.365 and 0.314 respectively). This indicated that among cells in the flushed femora, which would include osteoblasts at different stages of differentiation as well as osteocytes, are some cells that retain normal responses of these genes to PTH.

Wingless (WNT)-signaling is important for osteoblast differentiation and bone formation, and PTH has been shown to stimulate WNT signaling by suppressing Dickopf1 (Dkk1) and sclerostin (Sost) expression in the

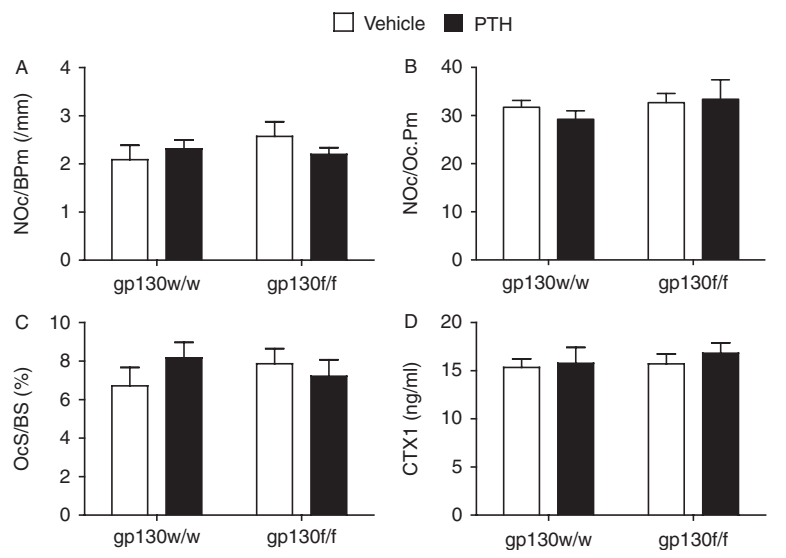

Figure 2

No effect of intermittent PTH on bone resorption. Male mice were treated with hPTH (1-34) at $30 \mu \mathrm{g} / \mathrm{kg}$ per day for 5 weeks. (A) Numbers of osteoclasts per unit bone perimeter (NOC/BPm), (B) numbers of osteoclasts per unit osteoclast perimeter ( $\mathrm{NOc} / \mathrm{OcPm}),(\mathrm{C})$ osteoclast surface per unit bone surface (OcS/BS) measured in the proximal tibial secondary spongiosa, and (D) serum levels of cross-linked C-terminal telopeptide of type 1 collagen (CTX1) in PTH and vehicle-treated Dmp1Cre.gp130w/w (gp130 $\mathrm{w} / \mathrm{w}$ ) and Dmp1Cre.gp130 f/f (gp130 f/f) mice. Scale bars are mean + s.E.M., $n=8-10$ per group.

Published by Bioscientifica Ltd. 

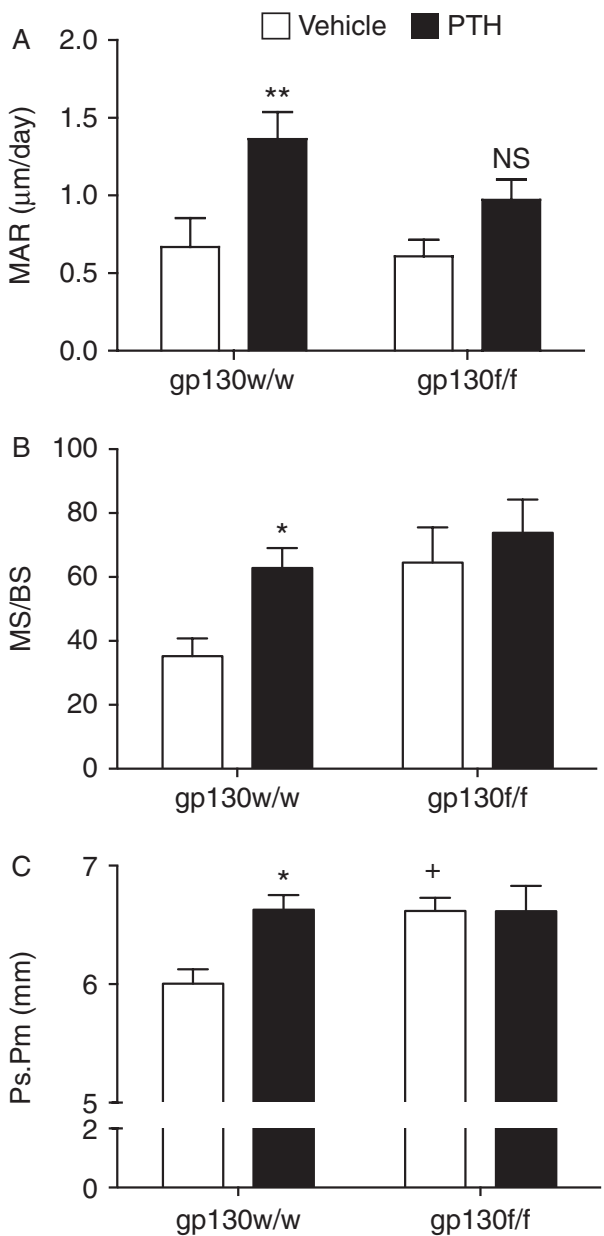

Figure 3

PTH effects on cortical bone are impaired in Dmp1Cre.gp130 fff mice. Male mice were treated with hPTH (1-34) at 30 $\mathrm{\mu g} / \mathrm{kg}$ per day for 5 weeks. (A) Tibial periosteal MAR (Ps.MAR), and (B) mineralizing surface per unit bone surface (MS/BS) in the tibial diaphysis, and (C) periosteal perimeter (Ps.Pm) of the femoral diaphysis in PTH and vehicle-treated Dmp1Cre.gp130 ${ }^{w / w}$ (gp130 w/w) and Dmp1Cre.gp130 f/f (gp130 f/f) mice. Scale bars are mean + s.E.M., $n=8-10$ per group. ${ }^{*} P \leq 0.01$, NS, $P>0.05$ (not statistically significant) in PTH-treated compared with genotype-matched vehicle-treated mice. ${ }^{+}, P<0.05$, vehicle-treated Dmp1Cre.gp $130^{f / f}$ compared with vehicle-treated Dmp1Cre.gp130 $\mathrm{w} / \mathrm{w}$.

osteoblast lineage (Keller \& Kneissel 2005, Yao et al. 2011). For this reason, we quantified mRNA levels of $D k k 1$ and Sost in flushed femurs. As expected, Dkk1 mRNA levels were significantly lower in PTH-treated Dmp1Cre.gp $130^{w / w}$ femurs compared with untreated mice. However, $D k k 1$ was not lower in femurs from PTH-treated Dmp1Cre.gp130f/f mice compared with controls (Fig. 4C). Sost mRNA levels were slightly, but not significantly, lowered in response to PTH in Dmp1Cre.gp130 ${ }^{w / w}$ femora. Dmp1Cre.gp130 f/f femora showed a lower level of Sost mRNA compared with vehicle-treated Dmp1Cre.gp130 w/w controls; with PTH treatment these mice showed a significant increase in Sost mRNA levels (Fig. 4D). These differences in the effects of PTH treatment on gene expression were significant by two-way ANOVA for both $D k k 1$ (interaction $P=0.01$ ) and Sost (interaction $P=0.003$ ). Thus, PTH treatment does not decrease WNT antagonist expression in Dmp1Cre.gp $130^{f / /}$ mice, implying that gp130 signaling in osteocytes is important for the PTH effect on WNT signaling inhibitors.

\section{Pth 1r expression is reduced in DMP1Cre.gp $130^{f / f}$ mice and gp130-deficient osteoblasts}

Since many effects of PTH were blocked in Dmp1Cre.gp130 f/f mice, we quantified Pth1r mRNA levels in flushed femurs from untreated 12-week-old Dmp1Cre.gp130 f/f and Dmp1Cre.gp130 ${ }^{w / w}$ mice. Surprisingly, Pth1r mRNA expression was $47 \%$ lower in Dmp1Cre.gp130 f/f compared with Dmp1Cre.gp130 w/w femurs ( $P=0.03$; Fig. $5 \mathrm{~A})$.

These findings were supported by in vitro data, where C57/BL6 and gp130 f/f calvarial osteoblasts were infected with lentiviral Cre-recombinase. In Cre-infected $g p 130^{f / f}$ osteoblasts, $g p 130$ was significantly lowered by $52 \%$, and Pth1r mRNA was $84 \%$ lower than in infected C57/BL6 cells
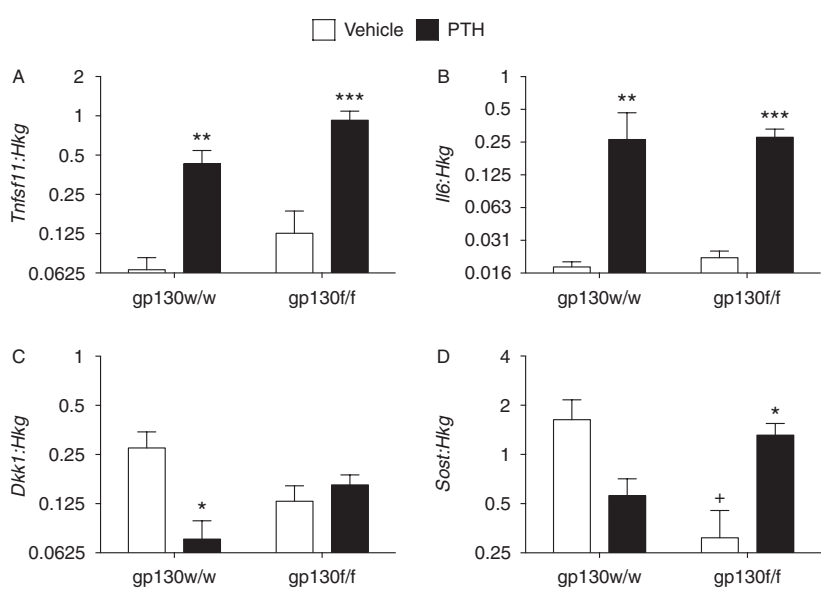

Figure 4

PTH effects on WNT-inhibitor, but not osteoclastogenic, mRNA levels are impaired in DMP1Cre.gp 130 f/f mice. RNA was isolated from femurs flushed of bone marrow and expression of PTH target genes was examined by relative quantitative PCR. Tnfs11 mRNA (A), 116 mRNA (B), Dkk1 mRNA (C) and Sost mRNA (D) in Dmp1Cre.gp130 w/w and Dmp1Cre.gp130 f/f mice treated for 5 weeks with PTH, collected $1 \mathrm{~h}$ after the final injection. All values are shown relative to housekeeping $(H \mathrm{~kg})$ - the geometric mean of hypoxanthine phosphoribosyltransferase 1 (Hprt1) and hydroxymethylbilane synthase (Hmbs). Scale bars are mean + S.E.M., $n=5-8$ bones per group, with mRNA prepared and analyzed separately. ${ }^{* * P} \leq 0.01$ and ${ }^{* * *} P \leq 0.001$, PTH-treated compared with genotype-matched vehicle-treated mice; ${ }^{+} P<0.05$, vehicle-treated $D m p 1 C r e . g p 130^{f / f}$ compared with vehicle-treated Dmp1Cre.gp130 w/w. 

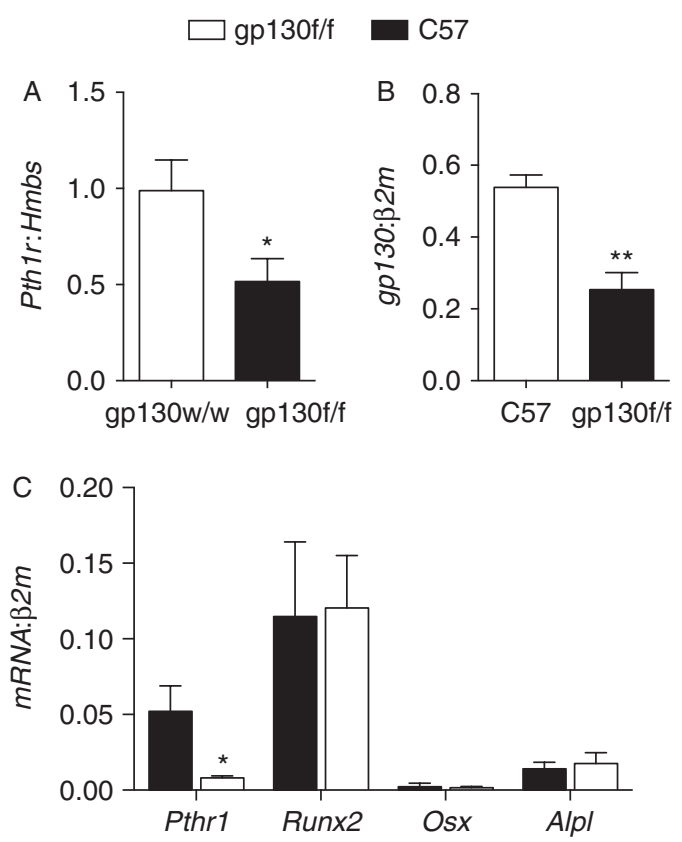

\section{Figure 5}

PTH1R expression is reduced in DMP1Cre.gp130 f/f mice and gp130 deficient cultured osteoblasts. (A) Pth1r mRNA quantified by qPCR in femurs flushed of bone marrow obtained from untreated 12-week-old Dmp1Cre.gp130 w/w and Dmp1Cre.gp130 f/f mice, normalized to Hmbs; $n=8$ samples per group. (B) gp130 (I/6st) and (C) Pth1r, Runx2, Osx and Alp/ mRNA levels in primary calvarial osteoblasts obtained from $g p 130^{f / f}$ or C57/BL6 WT neonates infected with lentiviral Cre-recombinase; levels are shown normalized to beta-2-microglobulin ( $B 2 m)$ ( $n=3$ biological replicates). ${ }^{*} P \leq 0.05$; $* * P \leq 0.01$, vs gp $130 \mathrm{w} / \mathrm{w}$ or C57/BL6.

(Fig. 5B and C). The mRNA levels of Runx2, Osx , and Alpl were not significantly altered by Cre-infection of $g p 130^{f / f}$ osteoblasts (Fig. 5C), consistent with previously published mRNA levels of these genes in the femora of Dmp1Cre.gp $130^{f / f}$ mice (Johnson et al. 2014b). This suggests that the cells of the osteoblast lineage require signals mediated by gp130 to maintain PTH1R expression, and that a lack of PTH1R in Dmp1Cre expressing cells is responsible for the reduced response to anabolic PTH treatment.

\section{Discussion}

This work demonstrates that PTH-induced osteoblast differentiation is dependent on gp130 expression in mature osteoblast lineage cells. gp130 is needed to maintain Pth1r expression in osteoblasts, and is required for PTH to suppress the WNT-antagonists Dkk1 and Sost. In contrast, gp130 expression by osteocytes is not required for PTH to stimulate mRNA levels of the pro-osteoclastogenic factors RANKL (Tnfsf11) and Il6 in bone.
The stimulatory effect of PTH on trabecular osteoblast numbers and mineralizing surface was completely ablated in Dmp1Cre.gp $130^{f / f}$ mice. This may, at least partly, be explained by the lack of a reduction in both WNT signaling inhibitors Sost and Dkk1 in response to PTH. WNT signaling stimulates osteoblast differentiation, and it has been postulated that this is one pathway through which PTH stimulates bone formation (Kulkarni et al. 2005), a hypothesis supported by impaired PTH responses in mice overexpressing sclerostin or Dkk1 (Guo et al. 2010, Kramer et al. 2010). PTH directly inhibits Sost via cAMP-PKA signaling (Keller \& Kneissel 2005). The IL-6 family cytokines also rapidly inhibit Sost, although the mechanism remains unknown (Walker et al. 2010). Whether the reduction in the effect of PTH on WNT signaling is entirely due to the reduced PTH1R expression or results from some dependence on gp130 cytokines by this same pathway in osteoblasts and osteocytes remains unclear.

In contrast to the effect on WNT-antagonists, both Dmp1Cre.gp130 $0^{w / w}$ and Dmp1Cre.gp130 f/f mice demonstrated increased femoral Tnfsf 11 and Il6 mRNA levels in response to PTH. Despite these increases in both genotypes, osteoclast numbers were unchanged, as we have previously reported with this low dose of intermittent PTH treatment (Takyar et al. 2013, Tonna et al. 2014), likely because the inductions of Tnfsf 11 and $I l 6$ are transient (Ma et al. 2001, Walker et al. 2012). IL-6 and RANKL are expressed by a wide range of cells in the bone, including osteoblast lineage cells as well as osteocytes (Lee \& Lorenzo 1999, Dai et al. 2006, Nakashima et al. 2011, Xiong et al. 2011), and cells within the bone marrow, including T-cells (Horwood et al. 1999, Hirano et al. 1986) and, in the case of IL-6, macrophages (Tosato et al. 1988). Although PTH has recently been suggested to directly promote RANKL expression in osteocytes (Xiong et al. 2011), our findings suggest that the major cellular targets that produce these pro-osteoclastogenic factors in response to PTH are not osteocytes. Notably, although PTH was unable to increase osteoblast numbers or mineralizing surface in the Dmp1Cre.gp $130^{f / f}$ mice, on those surfaces where double calcein labels were incorporated into the bone matrix, the distance between them (MAR) was significantly greater in PTH-treated mice, regardless of genotype. This suggests that bone-forming osteoblasts in Dmp1Cre.gp130//f mice retain sufficient PTHR expression to respond to PTH with increased matrix production. Since marrow was flushed from the femora, and Pth1r levels were dramatically reduced in undifferentiated cultured Cre-expressing cells, we suggest that the

Published by Bioscientifica Ltd. 
key PTH-responsive cells producing RANKL and IL-6 in this model are less differentiated osteoblasts, not expressing DMP1Cre, on the bone surface.

Pth1r mRNA was lower in cortical bone of Dmp1Cre.gp130 f/f mice compared with littermate controls, an effect that was reproduced when gp130 was deleted in cultured primary calvarial osteoblasts. There are two ways to understand this: firstly, as osteoblast differentiation is impaired in the Dmp1Cre.gp $130^{f / f}$ mice (Johnson et al. 2014b) and PTH1R expression in the osteoblast lineage is higher in more mature osteoblasts (Allan et al. 2003, 2008, Balic et al. 2010), there may be fewer mature PTH1R-expressing osteoblasts present within the bone of these mice. Another interpretation is that gp130 is needed to maintain the expression of PTH1R in the osteoblast lineage. This latter hypothesis is supported by our in vitro data, as we observed that a reduction in gp130 by about $50 \%$ in calvarial osteoblasts cultured in vitro reduced Pth1r mRNA levels by nearly $80 \%$. This further suggests that, as well as maintaining PTH1R levels in the osteocyte, gp130 may maintain PTH1R expression throughout the osteoblast lineage.

Although Pth1r levels were low in the femora of Dmp1Cre.gp130 f/f mice, their phenotype is strikingly different to mice with a conditional deletion of Pth1r in osteocytes (Ocy-PPRKO), generated using the same Dmp1Cre (Saini et al. 2013). Ocy-PPRKO mice showed a greater trabecular bone mass than controls, with no significant alteration in osteoblast numbers, indicating that the underlying cause of bone fragility in the Dmp1Cre.gp 130 f/f mice is not simply low PTH1R expression in the osteocyte. As observed in Dmp1Cre.gp130 f/f mice, Ocy-PPRKO mice failed to reduce Sost in response to PTH treatment. However, in direct contrast to Dmp1Cre.gp130 f/f mice, Ocy-PPRKO mice lacked a Tnfsf11 in response to PTH. This suggests that the Dkk1/Sost and Tnfsf11/Il6st responses to PTH occur in different cell populations, and it is only the former that is affected by Dmp1Cre-mediated gp130 deletion. Alternatively, the $D k k 1 /$ Sost induction may require a higher level of PTH1R expression than the Tnfsf11/Il6st response; the low level of PTH1R expression in the Dmp1Cre.gp130 f/f mice may be sufficient for the latter.

In addition to mediating the response of osteoblasts to exogenous PTH treatment, PTH1R also acts as a receptor for PTH-related protein (PTHrP). Although first identified as the mediator of humoral hypercalcemia of malignancy (Suva et al. 1987), PTHrP is also produced by the osteoblast lineage (Kartsogiannis et al. 1997). This local PTHrP production is essential for normal osteoblast differentiation, as indicated by studies of an osteoblastlineage PTHrP-null mice (Miao et al. 2005). This suggests that basal defects in osteoblast differentiation in our model lacking gp130 in osteocytes may relate specifically to a lack of PTHrP signal. Notably, and in direct contrast to our model, the osteoblast-lineage knockout of PTHrP also exhibited a significant impairment in osteoclastogenesis (Miao et al. 2005), a finding that may relate to the difference in the gene-driving expression of the Cre-recombinase. The Pthr $p^{f / f}$ deletion was driven by the Col2.3Cre, which would delete expression in osteocytes, but also in less mature osteoblasts than the Dmp1Cre that we have used. Again, this suggests that the PTH-induced expression of RANKL is likely to occur in less mature osteoblasts.

In conclusion, in addition to the recently described role of osteocytic gp130 in maintaining bone formation and strength (Johnson et al. 2014b), the current study has revealed a new role for gp130 in the osteoblast lineage in bone: it is needed to maintain PTH1R expression and to increase osteoblast numbers in response to anabolic PTH treatment.

\section{Declaration of interest}

The authors declare that there is no conflict of interest that could be perceived as prejudicing the impartiality of the research reported.

\section{Funding}

This work was supported by a National Health and Medical Research Council (Australia) Project Grant 1002728. N A S is supported by a National Health and Medical Research Council (Australia) Senior Research Fellowship. T S is supported by a Senior Research Fellowship from the Norwegian Cancer Society (project number 538153). The Victorian State Government Operational Infrastructure Support Scheme provides support to St. Vincent's Institute of Medical Research.

\section{Author contributions}

N A S and T S designed the study; T S, R W J, N E M, I J P, and P W M H conducted the study and made data collection; T S, R W J, N E M, I J P, P W M $\mathrm{H}$, and N A S analyzed data; T S, R W J, N E M, I J P, PW M H, T J M, and N A S interpreted data; T S and N A S drafted manuscript; T S, N A S, R W J, and $T J M$ revised the manuscript content. All authors approved the final version of manuscript. N A S takes responsibility for the integrity of the data analysis.

\section{Acknowledgements}

The authors thank Joshua Johnson for technical expertise in the processing of bone samples for histomorphometric analysis, Dr Stephen Tonna for advice on Lentiviral Cre infection, and Dr Nicole Walsh for design of the Dkk1 primers.

Published by Bioscientifica Ltd. 


\section{References}

Allan EH, Ho PW, Umezawa A, Hata J, Makishima F, Gillespie MT \& Martin TJ 2003 Differentiation potential of a mouse bone marrow stromal cell line. Journal of Cellular Biochemistry 90 158-169. (doi:10.1002/jcb. 10614)

Allan EH, Hausler KD, Wei T, Gooi JH, Quinn JM, Crimeen-Irwin B, Pompolo S, Sims NA, Gillespie MT, Onyia JE et al. 2008 EphrinB2 regulation by PTH and PTHrP revealed by molecular profiling in differentiating osteoblasts. Journal of Bone and Mineral Research $\mathbf{2 3}$ 1170-1181. (doi:10.1359/jbmr.080324)

Balic A, Aguila HL \& Mina M 2010 Identification of cells at early and late stages of polarization during odontoblast differentiation using pOBCol3.6GFP and pOBCol2.3GFP transgenic mice. Bone 47 948-958. (doi:10.1016/j.bone.2010.08.009)

Bellido T, Ali AA, Gubrij I, Plotkin LI, Fu Q, O’Brien CA, Manolagas SC \& Jilka RL 2005 Chronic elevation of parathyroid hormone in mice reduces expression of sclerostin by osteocytes: a novel mechanism for hormonal control of osteoblastogenesis. Endocrinology 146 4577-4583. (doi:10.1210/en.2005-0239)

Betz UA, Bloch W, van den Broek M, Yoshida K, Taga T, Kishimoto T, Addicks K, Rajewsky K \& Muller W 1998 Postnatally induced inactivation of gp130 in mice results in neurological, cardiac, hematopoietic, immunological, hepatic, and pulmonary defects. Journal of Experimental Medicine 188 1955-1965. (doi:10.1084/jem.188. 10.1955)

Cornish J, Callon K, King A, Edgar S \& Reid IR 1993 The effect of leukemia inhibitory factor on bone in vivo. Endocrinology 132 1359-1366. (doi:10. 1210/endo.132.3.8440191)

Dai JC, He P, Chen X \& Greenfield EM 2006 TNF $\alpha$ and PTH utilize distinct mechanisms to induce IL-6 and RANKL expression with markedly different kinetics. Bone 38 509-520. (doi:10.1016/j.bone.2005.10.007)

Dobnig H \& Turner RT 1995 Evidence that intermittent treatment with parathyroid hormone increases bone formation in adult rats by activation of bone lining cells. Endocrinology 136 3632-3638. (doi:10.1210/endo.136.8.7628403)

Greenfield EM, Shaw SM, Gornik SA \& Banks MA 1995 Adenyl cyclase and interleukin 6 are downstream effectors of parathyroid hormone resulting in stimulation of bone resorption. Journal of Clinical Investigation 96 1238-1244. (doi:10.1172/JCI118157)

Greenfield EM, Horowitz MC \& Lavish SA 1996 Stimulation by parathyroid hormone of interleukin- 6 and leukemia inhibitory factor expression in osteoblasts is an immediate-early gene response induced by cAMP signal transduction. Journal of Biological Chemistry 271 10984-10989. (doi:10.1074/jbc.271.18.10984)

Guo J, Liu M, Yang D, Bouxsein ML, Saito H, Galvin RJ, Kuhstoss SA, Thomas CC, Schipani E, Baron R et al. 2010 Suppression of Wnt signaling by Dkk1 attenuates PTH-mediated stromal cell response and new bone formation. Cell Metabolism 11 161-171. (doi:10.1016/j.cmet. 2009.12.007)

Hirano T, Yasukawa K, Harada H, Taga T, Watanabe Y, Matsuda T, Kashiwamura S-I, Nakajima K, Koyama K, Iwamatsu A et al. 1986 Complementary DNA for a novel human interleukin (BSF-2) that induces B lymphocytes to produce immunoglobulin. Nature 324 73-76. (doi:10.1038/324073a0)

Hodsman AB, Bauer DC, Dempster DW, Dian L, Hanley DA, Harris ST, Kendler DL, McClung MR, Miller PD, Olszynski WP et al. 2005 Parathyroid hormone and teriparatide for the treatment of osteoporosis: a review of the evidence and suggested guidelines for its use. Endocrine Reviews 26 688-703. (doi:10.1210/er.2004-0006)

Horwood NJ, Kartsogiannis V, Quinn JM, Romas E, Martin TJ \& Gillespie MT 1999 Activated T lymphocytes support osteoclast formation in vitro. Biochemical and Biophysical Research Communications 265 144-150. (doi:10.1006/bbrc.1999.1623)
Jilka RL, Weinstein RS, Bellido T, Roberson P, Parfitt AM \& Manolagas SC 1999 Increased bone formation by prevention of osteoblast apoptosis with parathyroid hormone. Journal of Clinical Investigation $\mathbf{1 0 4}$ 439-446. (doi:10.1172/JCI6610)

Johnson RW, White JD, Walker EC, Martin TJ \& Sims NA 2014a Myokines (muscle-derived cytokines and chemokines) including ciliary neurotrophic factor (CNTF) inhibit osteoblast differentiation. Bone 64C 47-56. (doi:10.1016/j.bone.2014.03.053)

Johnson RW, Brennan HJ, Vrahnas C, Poulton IJ, McGregor NE, Standal T, Walker EC, Koh TT, Nguyen H, Walsh NC et al. 2014b The primary function of gp130 signaling in osteoblasts is to maintain bone formation and strength, rather than promote osteoclast formation. Journal of Bone and Mineral Research 29 1492-1505. (doi:10.1002/jbmr.2159)

Kartsogiannis V, Moseley J, McKelvie B, Chou ST, Hards DK, Ng KW, Martin TJ \& Zhou H 1997 Temporal expression of PTHrP during endochondral bone formation in mouse and intramembranous bone formation in an in vivo rabbit model. Bone 21 385-392. (doi:10.1016/S87563282(97)00180-4)

Keller H \& Kneissel M 2005 SOST is a target gene for PTH in bone. Bone 37 148-158. (doi:10.1016/j.bone.2005.03.018)

Khosla S, Westendorf JJ \& Oursler MJ 2008 Building bone to reverse osteoporosis and repair fractures. Journal of Clinical Investigation 118 421-428. (doi:10.1172/JCI33612)

Kim SW, Pajevic PD, Selig M, Barry KJ, Yang JY, Shin CS, Baek WY, Kim JE \& Kronenberg HM 2012 Intermittent parathyroid hormone administration converts quiescent lining cells to active osteoblasts. Journal of Bone and Mineral Research 27 2075-2084. (doi:10.1002/jbmr.1665)

Kramer I, Loots GG, Studer A, Keller H \& Kneissel M 2010 Parathyroid hormone (PTH)-induced bone gain is blunted in SOST overexpressing and deficient mice. Journal of Bone and Mineral Research 25 178-189. (doi:10.1359/jbmr.090730)

Kulkarni NH, Halladay DL, Miles RR, Gilbert LM, Frolik CA, Galvin RJ, Martin TJ, Gillespie MT \& Onyia JE 2005 Effects of parathyroid hormone on Wnt signaling pathway in bone. Journal of Cellular Biochemistry 95 1178-1190. (doi:10.1002/jcb.20506)

Lee SK \& Lorenzo JA 1999 Parathyroid hormone stimulates TRANCE and inhibits osteoprotegerin messenger ribonucleic acid expression in murine bone marrow cultures: correlation with osteoclast-like cell formation. Endocrinology 140 3552-3561. (doi:10.1210/endo.140.8. 6887)

Lindsay R, Zhou H, Cosman F, Nieves J, Dempster DW \& Hodsman AB 2007 Effects of a one-month treatment with PTH(1-34) on bone formation on cancellous, endocortical, and periosteal surfaces of the human ilium. Journal of Bone and Mineral Research 22 495-502. (doi:10.1359/ jbmr.070104)

Lu Y, Xie Y, Zhang S, Dusevich V, Bonewald LF \& Feng JQ 2007 DMP1targeted Cre expression in odontoblasts and osteocytes. Journal of Dental Research 86 320-325. (doi:10.1177/154405910708600404)

Ma YL, Cain RL, Halladay DL, Yang X, Zeng Q, Miles RR, Chandrasekhar S, Martin TJ \& Onyia JE 2001 Catabolic effects of continuous human PTH (1-38) in vivo is associated with sustained stimulation of RANKL and inhibition of osteoprotegerin and gene-associated bone formation. Endocrinology 142 4047-4054. (doi:10.1210/endo.142.9.8356)

McGregor NE, Poulton IJ, Walker EC, Pompolo S, Quinn JM, Martin TJ \& Sims NA 2010 Ciliary neurotrophic factor inhibits bone formation and plays a sex-specific role in bone growth and remodeling. Calcified Tissue International 86 261-270. (doi:10.1007/s00223-010-9337-4)

Miao D, He B, Jiang Y, Kobayashi T, Soroceanu MA, Zhao J, Su H, Tong X, Amizuka N, Gupta A et al. 2005 Osteoblast-derived PTHrP is a potent endogenous bone anabolic agent that modifies the therapeutic efficacy of administered PTH 1-34. Journal of Clinical Investigation 115 2402-2411. (doi:10.1172/JCI24918)

Nakashima T, Hayashi M, Fukunaga T, Kurata K, Oh-Hora M, Feng JQ, Bonewald LF, Kodama T, Wutz A, Wagner EF et al. 2011 Evidence for

Published by Bioscientifica Ltd. 
osteocyte regulation of bone homeostasis through RANKL expression. Nature Medicine 17 1231-1234. (doi:10.1038/nm.2452)

Neer RM, Arnaud CD, Zanchetta JR, Prince R, Gaich GA, Reginster JY, Hodsman AB, Eriksen EF, Ish-Shalom S, Genant HK et al. 2001 Effect of parathyroid hormone (1-34) on fractures and bone mineral density in postmenopausal women with osteoporosis. New England Journal of Medicine 344 1434-1441. (doi:10.1056/NEJM200105103441904)

O’Brien CA, Gubrij I, Lin SC, Saylors RL \& Manolagas SC 1999 STAT3 activation in stromal/osteoblastic cells is required for induction of the receptor activator of NF- $\mathrm{B}$ ligand and stimulation of osteoclastogenesis by gp130-utilizing cytokines or interleukin-1 but not 1,25dihydroxyvitamin D3 or parathyroid hormone. Journal of Biological Chemistry 274 19301-19308. (doi:10.1074/jbc.274.27.19301)

Palmqvist P, Persson E, Conaway HH \& Lerner UH 2002 IL-6, leukemia inhibitory factor, and oncostatin $\mathrm{M}$ stimulate bone resorption and regulate the expression of receptor activator of NF-ка B ligand, osteoprotegerin, and receptor activator of NF-ка B in mouse calvariae. Journal of Immunology 169 3353-3362. (doi:10.4049/jimmunol.169. 6.3353)

Parfitt AM, Drezner MK, Glorieux FH, Kanis JA, Malluche H, Meunier PJ, Ott SM \& Recker RR 1987 Bone histomorphometry: standardization of nomenclature, symbols, and units. Report of the ASBMR Histomorphometry Nomenclature Committee. Journal of Bone and Mineral Research 2 595-610. (doi:10.1002/jbmr.5650020617)

Reeve J, Meunier PJ, Parsons JA, Bernat M, Bijvoet OL, Courpron P, Edouard C, Klenerman L, Neer RM, Renier JC et al. 1980 Anabolic effect of human parathyroid hormone fragment on trabecular bone in involutional osteoporosis: a multicentre trial. BMJ 280 1340-1344. (doi:10.1136/ bmj.280.6228.1340)

Richards CD, Langdon C, Deschamps P, Pennica D \& Shaughnessy SG 2000 Stimulation of osteoclast differentiation in vitro by mouse oncostatin $\mathrm{M}$, leukaemia inhibitory factor, cardiotrophin-1 and interleukin 6: synergy with dexamethasone. Cytokine 12 613-621. (doi:10.1006/cyto.1999. 0635)

Romas E, Udagawa N, Zhou H, Tamura T, Saito M, Taga T, Hilton DJ, Suda T, $\mathrm{Ng}$ KW \& Martin TJ 1996 The role of gp130-mediated signals in osteoclast development: regulation of interleukin 11 production by osteoblasts and distribution of its receptor in bone marrow cultures. Journal of Experimental Medicine 183 2581-2591. (doi:10.1084/jem.183.6.2581)

Saini V, Marengi DA, Barry KJ, Fulzele KS, Heiden E, Liu X, Dedic C, Maeda A, Lotinun S, Baron R et al. 2013 Parathyroid hormone (PTH)/PTH-related peptide type 1 receptor (PPR) signaling in osteocytes regulates anabolic and catabolic skeletal responses to PTH. Journal of Biological Chemistry 288 20122-20134. (doi:10.1074/jbc.M112.441360)

Sims NA \& Walsh NC 2010 GP130 cytokines and bone remodelling in health and disease. BMB Reports 43 513-523. (doi:10.5483/BMBRep. 2010.43.8.513)

Sims NA, Brennan K, Spaliviero J, Handelsman DJ \& Seibel MJ 2006 Perinatal testosterone surge is required for normal adult bone size but not for normal bone remodeling. American Journal of Physiology. Endocrinology and Metabolism 290 E456-E462. (doi:10.1152/ajpendo. 00311.2005)
Suva LJ, Winslow GA, Wettenhall RE, Hammonds RG, Moseley JM, Diefenbach-Jagger H, Rodda CP, Kemp BE, Rodriguez H, Chen EY et al. 1987 A parathyroid hormone-related protein implicated in malignant hypercalcemia: cloning and expression. Science 237 893-896. (doi:10.1126/science.3616618)

Takyar FM, Tonna S, Ho PW, Crimeen-Irwin B, Baker EK, Martin TJ \& Sims NA 2013 EphrinB2/EphB4 inhibition in the osteoblast lineage modifies the anabolic response to parathyroid hormone. Journal of Bone and Mineral Research 28 912-925. (doi:10.1002/jbmr.1820)

Tamura T, Udagawa N, Takahashi N, Miyaura C, Tanaka S, Yamada Y, Koishihara Y, Ohsugi Y, Kumaki K, Taga T et al. 1993 Soluble interleukin-6 receptor triggers osteoclast formation by interleukin 6 . PNAS 90 11924-11928. (doi:10.1073/pnas.90.24.11924)

Tonna S, Takyar FM, Vrahnas C, Crimeen-Irwin B, Ho PW, Poulton IJ, Brennan HJ, McGregor NE, Allan EH \& Nguyen H 2014 EphrinB2 signaling in osteoblasts promotes bone mineralization by preventing apoptosis. FASEB Journal [in press]. (doi:10.1096/fj.14-254300)

Torreggiani E, Matthews BG, Pejda S, Matic I, Horowitz MC, Grcevic D \& Kalajzic I 2013 Preosteocytes/osteocytes have the potential to dedifferentiate becoming a source of osteoblasts. PLOS ONE 8 e75204. (doi:10.1371/journal.pone.0075204)

Tosato G, Seamon KB, Goldman ND, Sehgal PB, May LT, Washington GC, Jones KD \& Pike SE 1988 Monocyte-derived human B-cell growth factor identified as interferon-beta 2 (BSF-2, IL-6). Science 239 502-504. (doi:10.1126/science.2829354)

Udagawa N, Takahashi N, Jimi E, Matsuzaki K, Tsurukai T, Itoh K, Nakagawa N, Yasuda H, Goto M, Tsuda E et al. 1999 Osteoblasts/stromal cells stimulate osteoclast activation through expression of osteoclast differentiation factor/RANKL but not macrophage colony-stimulating factor: receptor activator of NF-ка B ligand. Bone 25 517-523. (doi:10.1016/S8756-3282(99)00210-0)

Walker EC, McGregor NE, Poulton IJ, Pompolo S, Allan EH, Quinn JM, Gillespie MT, Martin TJ \& Sims NA 2008 Cardiotrophin-1 is an osteoclast-derived stimulus of bone formation required for normal bone remodeling. Journal of Bone and Mineral Research 23 2025-2032. (doi:10.1359/jbmr.080706)

Walker EC, McGregor NE, Poulton IJ, Solano M, Pompolo S, Fernandes TJ, Constable MJ, Nicholson GC, Zhang JG, Nicola NA et al. 2010 Oncostatin $\mathrm{M}$ promotes bone formation independently of resorption when signaling through leukemia inhibitory factor receptor in mice. Journal of Clinical Investigation 120 582-592. (doi:10.1172/JCI40568)

Walker EC, Poulton IJ, McGregor NE, Ho PW, Allan EH, Quach JM, Martin TJ \& Sims NA 2012 Sustained RANKL response to parathyroid hormone in oncostatin $\mathrm{M}$ receptor-deficient osteoblasts converts anabolic treatment to a catabolic effect in vivo. Journal of Bone and Mineral Research 27 902-912. (doi:10.1002/jbmr.1506)

Xiong J, Onal M, Jilka RL, Weinstein RS, Manolagas SC \& O’Brien CA 2011 Matrix-embedded cells control osteoclast formation. Nature Medicine $\mathbf{1 7}$ 1235-1241. (doi:10.1038/nm.2448)

Yao GQ, Wu JJ, Troiano N \& Insogna K 2011 Targeted overexpression of Dkk1 in osteoblasts reduces bone mass but does not impair the anabolic response to intermittent PTH treatment in mice. Journal of Bone and Mineral Metabolism 29 141-148. (doi:10.1007/s00774-010-0202-3)

Received in final form 19 August 2014

Accepted 15 September 2014

Accepted Preprint published online 16 September 2014 http://joe.endocrinology-journals.org DOI: 10.1530/JOE-14-0424
() 2014 Society for Endocrinology Printed in Great Britain 